\title{
Perceptual independence of pitch and loudness in a signal detection experiment: A processing model for $2 \mathrm{ATFC}(21 \mathrm{FC})$ experiments
}

\author{
MICHAEL ZAGORSKI \\ Memorial University of Newfoundland, St. John's, Newfoundland, Canada
}

\begin{abstract}
The two-alternative temporal forced choice (2ATFC) experiment is used to measure the relative detectabilities of a frequency change, an amplitude increase, or both together. Subjects' performance is best when both (redundant) cues are available. This improvement is fit better by a decision threshold model than by an information integration model. Since decision processes that can lead to the decision threshold prediction in (2ATFC) experiments are not obvious, an information processing model which does is proposed. The model makes additional predictions which fit the results of an experiment which sometimes deletes information from the first or the second observation interval. The model is not consistent with the signal detection theory interpretation of the 2ATFC experiment, and these results call into question that interpretation. It is concluded that pitch and loudness are perceptually independent for the 2ATFC experiment.
\end{abstract}

This study is based upon the description of sinusoidal tones as points within a two-dimensional, pitch-loudness space. The assumption underlying the application of this spatial model is that the distance separating a pair of stimuli on a unidimensional continuum represents their psychological dissimilarity. Thus the presentation of sinusoidal tones as points in a two-dimensional space may be assumed to indicate that the overall similarity of a pair of sinusoidal tones, differing on two dimensions, such as loudness and pitch, is related to their two-dimensional Euclidean distance.

Discriminability of stimuli may be interpreted as a measure of their similarity. The question then becomes, does discriminability display some of the properties of Euclidean space? According to the spatial model, a pair of tones which differ in both pitch and loudness should be more discriminable than a pair of tones that have the same pitch differences but identical loudness, and vice versa. It can also be argued, however, that the subject can attend to only one dimension at $i$ time, so the question is raised as to what strategies the subject can, and does, employ in a particular task.

Since the two-alternative temporal forced choice task is regarded as a suspension measure of discriminability (because, according to the theory of signal detectability, it depends neither on criterion

This research was supported in part by National Science Foundation Grant NSF GB 5270 to Dr. H. Lindman and by Public Health Service Grant MH-16817 to Dr. F. Restle while the author was a mathematical psychology training fellow under the supervision of Dr. Restle. The instrumentation was carried out in consultation with Dr. D. Robinson. The preparation of the manuscript was supported in part by National Research Council of Canada Grant A9804 to the author. nor on the distribution of sensory effects), it was employed in the study. However, Treisman and Leshowitz (1969) have suggested another information processing strategy for the task. Thus, the way subjects appear to combine pitch difference and loudness difference in this task may depend on the strategy or decision process he uses to perform the task. Therefore, this study is as much concerned with information processing in signal detection as it is with multidimensional information processing.

Some investigators suggest that for stimuli which vary on perceptually distinct dimensions. a spatial representation of stimulus similarity may be impossible. But they all suggest that the representation of similarity as a distance is appropriate for stimuli such as sinusoidal tones.

Shepard (1964) attempted to apply the spatial model to a task which used stimuli consisting of circles with a radius drawn in. The circles had various diameters and the radii various inclinations, thus making the stimulus set two-dimensional. The subject's task was to pick the pair of stimuli, from a set of pairs, that was most similar. The first stimulus of the set was a reference, and the second stimulus differed from the reference on at least one dimension. His detailed examination of the data revealed that the subjects were attending to one dimension or the other, thereby not using the two dimensions to create their overall impression of similarity, if indeed they had one. It was observed that subjects clearly perceived that the stimuli consisted of two perceptually distinct parts, a circle and a radius drawn in. He tentatively concluded that for highly analyzable stimuli, such as the circles with the radii drawn in, the representation of stimulus similarity as a distance in the multidimensional space was not appropriate. Shepard 
concluded that his data were consistent with Torgerson's (1958) conjecture that a Euclidean distance function would probably work for stimuli which "tend to be perceived as homogenous unanalyzable wholes such as sinusoidal tones."

Hyman and Well (1967) found that a Euclidean metric worked for the judged similarity of color patches, but not for geometric forms, including stimuli similar to those used by Shepard in 1904 . These findings supported Torgerson's conjecture that a Euclidean metric would represent the psychological similarity of sinusoidal tones.

Zagorski (Note 1) tested this hypothesis using a two-dimensional bisection task, but found that observers did not, in fact, use both the pitch and loudness in adjusting a variable stimulus so that it was equally dissimilar from two fixed stimuli. He found that subjects attended to either one dimension or the other and never seemed to combine the dimensions into an overall dissimilarity judgment.

Corcoran (1967) found that subjects used both pitch and loudness in the recognition of one particular acoustic stimulus called a target, but found that his data fit a decision threshold model (Green \& Swets, 1966) in which subjects arrive at separate decisions on each of the independent dimensions rather than combining the information and then making a decision on the combined information. Thus he finds that observers do not integrate pitch and loudness into a single dimension of information. However, his experiment involved reasonably discriminable stimuli in a two-to-four-dimensional recognition task. The errors in this experiment depended perhaps more on the memory load than on the sensory load.

The present study looks at how subjects combine the two acoustic dimensions of frequency and intensity in a signal detection task, in which errors are more likely to be due to limitations of sensory processes than memory processes.

Although they were not interested in twodimensional information processing, Harris, Pickler, Hoffman, and Ehmer (1958) did an experiment which seems to bear on this question, but they used an ABX procedure in which the subject's task was to listen to two reference tones (A and $B$ ) and report whether they thought a test tone $(X)$ was more like the first or the second reference tone. The ABX procedure, therefore, requires the subject to remember the $A$ and $\mathrm{B}$ signals for comparison with $\mathrm{X}$. In the experiment reported here, the subject merely had to notice a deviation from a reference.

The ABX procedure involving two dimensions of discriminability is rather like a concept identification experiment in which the subject must not only select the dimension but also discriminate highly confusable values on the dimensions. Thus, when the two cues are completely correlated, as in the Harris et al experiment, he can "solve" the problem by using whichever cue is perceptually available at the time. In the problem-solving literature, the decision threshold is shown to apply; that is, the subject can use one dimension, the other dimension, or both. We would expect, therefore, the decision threshold model to apply to two-dimensional signals in the ABX procedure. In fact, Pollack (1961) analyzed the data from Harris et al from the point of view of the several decision models and found a decision threshold model to fit best.

A previous study by Jauhiainen, Häkkiene, Lindroos, and Raij (1967) attempted to answer a similar question by using threshold measurements where the threshold was defined as being that difference which the subject could perceive $60 \%$ of the time. Their threshold measurements satisfied neither the decision threshold model nor a Euclidean two-dimensional model proposed by Reenpää (1961).

Since threshold measures are subject to a number of failings, such as criterion shifts (Green \& Swets, 1966), the study reported here uses the signal detection measure, probability correct, $P(C)$, in a two-alternative temporal forced-choice (2ATFC) task.

The task does not have a completely unambiguous interpretation; Treisman and Leshowitz (1969) suggest two strategies that subjects may use to perform the 2ATFC task-differencing and double detection. In the differencing strategy, the subject compares the first interval to the second and makes the decision on this basis. In the double detection strategy, the subject attempts to detect the signal in each interval; if he makes no detections or two detections, he guesses, and if he makes one detection, he makes the appropriate response. The differencing strategy leads to information integration prediction discussed below, and the double detection strategy leads to the decision threshold prediction. Thus, studying the way subjects combine dimensions can elucidate the nature of the strategies used.

\section{EXPERIMENT I}

\section{Method}

The subjects were two undergraduates paid $\$ 1.75 / \mathrm{h}$. Their task was to indicate in which of two observation intervals a change (in amplitude or frequency or both) of a continuing sinusoidal pedestal occurred.

As is shown in Figure 1, the beginning of each trial was marked by a warning light $(0.1 \mathrm{sec})$. Each trial consisted of the following sequence of events (in addition to the warning lights). The reference tone sounded $(0.5 \mathrm{sec})$, the first observation interval was presented $(0.2 \mathrm{sec})$, the reference tone sounded again $(0.5 \mathrm{sec})$, the second observation interval was presented $(0.2 \mathrm{sec})$, and finally the reference tone sounded during the response interval $(1.1 \mathrm{sec})$.

A band-limited $(250 \mathrm{~Hz}$ to $2 \mathrm{kHz})$ white noise formed a continuous background throughout the trial. Each observation interval was acoustically isolated by a $0.1-\mathrm{sec}$ "silent" period in which the sinusoids were switched off at the beginning and on at the end of each silent period (10-msec rise and decay time). The silent period, together with filtered (Kronhite electronic filter-10- $\mathrm{Hz}$ passband centered at $1,005 \mathrm{~Hz}$ ) switching, allowed signal intensity or signal frequency to be changed while minimizing the audibility and discriminability of transients. 


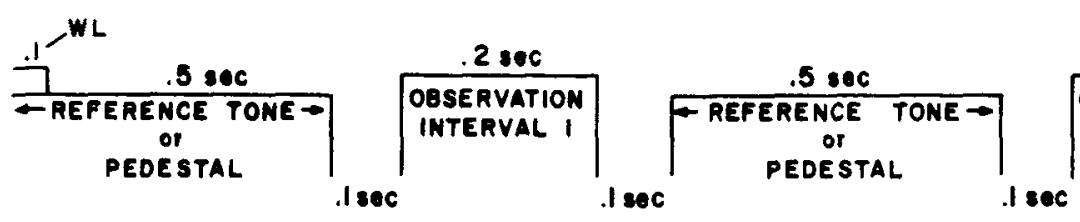

$.2 \sec$ O8SERVATION INTERVAL 2 -REFERENCE TONE -
Or
PEDESTAL
ASOC $1.1 \mathrm{sec}$

$2.8 \sec$

Figure 1. Sequence of events in the two-alternative temporal forced choice signal detection paradigm.

The observation intervals were marked with lights over the appropriate response keys; a light over the left key turned on during the first observation interval and over the right key during the second observation interval. The warning light was located at the top of the response panel.

In one condition, $\Delta \mathrm{l}$, the subject's task was to indicate in which of the two observation intervals he could detect the change when the intensity was different; in another condition, $\Delta f$, it was the frequency difference he had to detect, and in the third condition, $\Delta f+\Delta I$, it was both.

The reference tone was a $1.000-\mathrm{Hz}$ sinusoid at $67 \mathrm{~dB}$ SPL (as estimated from the TDH-39 earphone calibrations). The "signals" or changes involved amplitude increments of $2 \mathrm{~dB}$ and frequency increments of $3.5,5$, and $11.5 \mathrm{~Hz}$, as shown in Table 1 . For the band-limited noise $10 \log E /$ No. was equal to 26 .

Subjects were run in blocks of 100 trials with a short rest of a minute or less between blocks. During the rest, subjects were told the number of trials for which they failed to enter their response on time and number of correct responses they made. They were given a longer rest after four or five blocks during which they were allowed to come out of the booth. After training, each subject ran a few blocks on each condition every day, subject to the vagaries of scheduling at the Indiana University Mathematical Psychology Laboratory. ${ }^{1}$

\section{Results and Discussion}

The probability of a correct response, $P(C)$, from each condition (estimated by averaging over all blocks except practice and warm-up blocks) for each subject and each condition is shown in the third column of Table 1. It can be seen that performance was better in $\Delta \mathrm{f}+\Delta \mathrm{I}$ condition where both cues were available.

One can partition the set of all possible outcomes in the following way: First, having the additional cue impairs performance:

$$
P(C) \Delta f+\Delta l \leqslant \min [P(C) \Delta I, P(C) \Delta f] .
$$

Second, the subjects performance in the two-cue situation is midway between his performance on each of the cues separately:

$$
\begin{aligned}
& \min [P(C) \Delta l, P(C) \Delta t] \leqslant P(C) \Delta f+\Delta I \\
& <\max \left[\mathrm{P}(\mathrm{C}) \Delta \mathrm{II}, \mathrm{P}(\mathrm{C})_{\Delta f}\right] \text {. }
\end{aligned}
$$

This is the expected result if the subject samples one cue on certain trials and the other on the remaining trials. Third, in the two-cue, $\Delta \mathrm{I}+\Delta \mathrm{f}$, situation, the subject may simply use the most valid cue:

$$
\mathrm{P}(\mathrm{C})_{\Delta \mathrm{f}+\Delta \mathrm{I}}=\max \left[\mathrm{P}(\mathrm{C})_{\Delta \mathrm{I}}, \mathrm{P}(\mathrm{C}) \Delta \mathrm{f}\right] .
$$

In the fourth general outcome set, the subjects' performance in the two-cue case may exceed his performance in each of the single cue conditions:

$$
\mathrm{P}(\mathrm{C})_{\Delta f}+\Delta \mathrm{I}>\max [\mathrm{P}(\mathrm{C}) \Delta \mathrm{I}, \mathrm{P}(\mathrm{C}) \Delta \mathrm{f}] \text {. }
$$

This last general outcome set contains two interesting points predicted by two important models. One is the point predicted by the decision threshold model, as mentioned above. In this model, the subject hypothetically makes a decision about the loudness cue and a decision about the pitch cue, and then combines these decisions according to a general rule, such as, "If I detect a pitch change or a loudness change, or both, I will decide that I had detected the signal." Thus the probability of detecting the combined cue, $P(D) \Delta f+\Delta I$, can be related to the probability of detecting each of the single cues separately. The probability of detecting the combined cue, $P(D) \Delta f+\Delta l$, is equal to the probability of detecting the loudness cue, $P(D) \Delta I$, plus the probability of detecting the pitch cue, $P(D) \Delta f$, minus the probability of detecting both:

$$
P(D)_{\Delta I}+\Delta f=P(D)_{\Delta I}+P(D)_{\Delta f}-P(D)_{\Delta I} \cdot P(D)_{\Delta f} .
$$

But the probability of detecting a cue is not equal to the probability of being correct when that cue is present, because the subject might make a correct guess. In order to relate these two probabilities, we need a further assumption. For simplicity, and because it fits, let us make the high threshold theory assumption, which is that the subject says a cue is present if he detected it and otherwise guesses correctly with a probability of 0.5 :

$$
\mathrm{P}(\mathrm{C})=\mathrm{P}(\mathrm{D})+0.5[1-\mathrm{P}(\mathrm{D})] \text {. }
$$

The guessing probability is 0.5 because he chooses each of the two alternatives with an equal probability. 
We assume there is no bias for choosing one interval or the other. (No bias appeared in the data.) Thus,

$$
\mathrm{P}(\mathrm{C}) \Delta \mathrm{f}+\Delta \mathrm{I}=\frac{\mathrm{P}(\mathrm{C}) \Delta \mathrm{I}+\mathrm{P}(\mathrm{C}) \Delta \mathrm{f}-\mathrm{P}(\mathrm{C}) \Delta \mathrm{I}(\mathrm{C}(\mathrm{C}) \Delta \mathrm{f}-0.5}{0.5}
$$

The second interesting point in this last general outcome set is that predicted by the information integration model in which subjects hypothetically combine the pitch information with the loudness information to estimate the likelihood that the two-cue signal was present and make a single decision based on combined information. (See Green \& Swets. 1966.) The predictions from this model are made by assuming that there is no response bias and the sensory distributions are almost normal. According to this model, the measures of $d^{\prime}$ for the three conditions are related in the following way:

$$
\left(\mathrm{d}^{\prime} \Delta \mathrm{f}+\Delta \mathrm{I}\right)^{2}=\left(\mathrm{d}^{\prime} \Delta \mathrm{I}\right)^{2}+\left(\mathrm{d}^{\prime} \Delta \mathrm{f}\right)^{2} .
$$

Table 1 shows the predictions from the decision threshold and information integration models along with the data from the experiment. The predictions are based on finding those parameters in Equations 7 and 8 which minimize the chi square between the predictions and the data. The chi squares were minimized by scanning the parameters and computing the chi square of parameters that give predicted values near the observed. The final scan involves step sizes that correspond to changes in the predicted $\mathrm{P}(\mathrm{C})$ of about .001 . Thus, for each model, the best set of $P(C)$ are chosen to within .001 . The chi squares for each subject and each model are shown in Table 1 in the box containing the predictions from each model and each subject. It can be seen that the high threshold version of the decision threshold model fits quite well and better than the information integration model.

The high threshold model has been amply falsified (cf. Green \& Swets, 1966). Therefore, we must express surprise (as did Pollack, 1961) that the high threshold version of decision theshold theory fits so well. However, Treisman and Leshowitz's (1969) double detection model can explain these results. We can only conclude that the subjects were using a decision process that gives the same results as the high threshold version of decision threshold theory.

It should be noted that the information integration model can fit these results exactly if one assumes that the sinusoids involved are not orthogonal. Tanner (1958) worked out this theory. His theory takes into account the correlation of sinusoids of different frequencies. For each subject and listening condition, a parameter. $\theta$. measures the orthogonality of every
Table 1

Predicted and Observed $P(C)$ by Subject and Condition

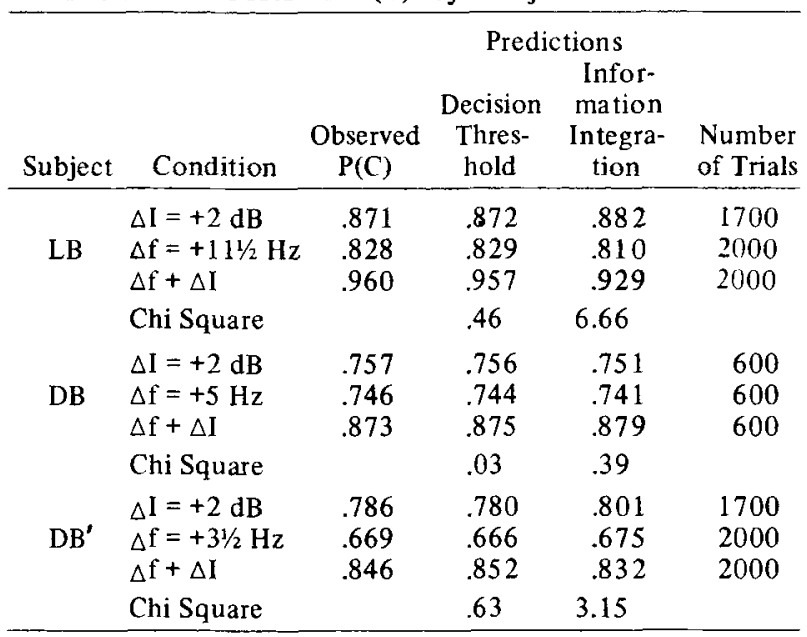

pair of sinusoids of different frequency. The experiment reported here was not designed to test this theory. By adjusting $\Theta$, the probability correct can be fit exactly for $\Delta \mathrm{I}, \Delta \mathrm{f}$, and $\Delta \mathrm{I}+\Delta \mathrm{f}$. Since $\Theta$ depends on the two frequencies involved, a proper test of the theory would use two or more levels of $\Delta \mathrm{I}$ for the $\Delta \mathrm{I}$ and $\Delta \mathrm{I}+\Delta \mathrm{f}$ conditions. Then the theory should fit, subject to the constraint that $\Theta$ is independent of $\Delta \mathbf{I}$. However, in view of the fact that the decision threshold model fits so well for all these data, including the subject that was run on two different $\Delta \mathrm{f}$, it can be argued that it is highly unlikely that the $\Delta \mathrm{fs}$ involved just happen to be associated with $\Theta$ that allow the decision threshold model to fit.

\section{AN INFORMATION PROCESSING MODEL}

Let us examine an information processing model which postulates high thresholds and predicts the decision threshold result of the first experiment. This model is put forward primarily as a heuristic. It specifies a reasonable sequential decision process which is consistent with the decision threshold notion. Simply put. it says that if the subject hears the signal in the first interval he doesn't listen to the second interval; he only listens to the second if he is not certain about the first.

The decision process is depicted in Figure 2. It shows that the subject may go into a detect state, D1, from the first observation interval, OI-1. Let us assume that the subject's criterion or threshold for going into a detect state is so high that he never goes into that state when the signal is absent, but that he has a finite probability of going into that state when the signal is present in the first observation interval as is the case with a normal Type 1 trial. In this high threshold model, if he goes into a detect state in the 


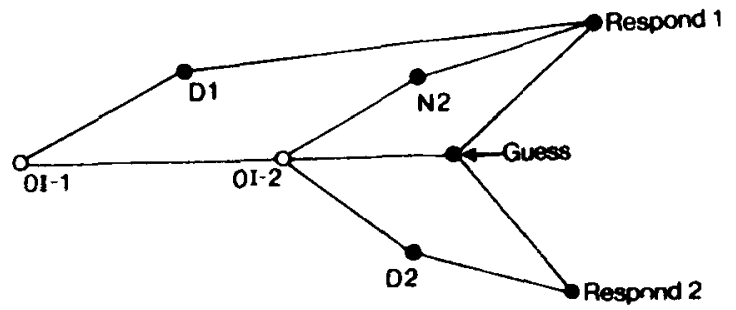

Figure 2. Proposed decision process for the 2ATFC experiment.

first observation interval, he responds automatically that it was a Type 1 trial. On the other hand, if he does not go into a detect state as a result of the first observation interval, he listens to the second observation interval, Ol-2, where he may go into a detect state, D2, a nondetect state, N2, or a guessing state. The nondetect state can be interpreted as the state he is in when he decides with a high degree of certainty that the signal was not in the observation interval. If he goes into D2, he responds 2 -that it was a Type 2 trial; if he goes into $\mathrm{N} 2$, he responds 1 ; if he goes into the guessing state, he guesses 1 or 2 .

In order to see if this model is consistent with the decision threshold fit observed in the first experiment and to derive independently testable predictions, let us write out the conditional probabilities associated with the model. Let $P(1 \mid 1)$ be the probability that the subject responds that the signal was in the first interval, given that it was in the first interval, $P(2 \mid 1)$ be the probability that the subject responds that it was in the second interval, given that it was in the first interval, $P(1 \mid 2)$ be the probability that the subject responds 1 , given that it was a 2 , and $P(2 \mid 2)$ be the probability that the subject says 2 , given that it was a 2.

He can correctly respond 1 , given that it was a 1 , by going into a detect state, D1, after OI-1, where he got the signal $\mathrm{S} 1$ or by going into a nondetect state, $\mathrm{N} 2$, after OI-2, or by doing neither and guessing correctly. This means that $P(1 \mid 1)$ is related to the probability of going into D1, given a signal in (S1), P(D1 $\mid S 1)$ and the probability of going into $\mathrm{N} 2$, given no signal in $2(\mathrm{~S} 2), \mathrm{P}(\mathrm{N} 2 \mid \overline{\mathrm{S}} 2)$ and the probability of guessing 1 , G1, as follows:

$$
\begin{aligned}
P(1 \mid 1)= & P(D|| S 1)+[1-P(D 1 \mid S 1)] \\
& \{P(2 \mid \bar{S} 2)+[1 \quad P(N 2 \mid S 2) \mid G 1\} .
\end{aligned}
$$

He says the signal occurred in 2 when it really occurred in 1 when he fails to go into a detect state in 1 and he fails to go into a nondetect state in 2 and guesses that it is a 2 (with probability G2); therefore:

$$
P(2 \mid 1)=\left[\begin{array}{ll}
P(D|| S 1)
\end{array}\right] \cdot[1 \quad P(N 2 \mid S 2)] G 2 \text {. (10) }
$$

He says that signal occurred in 2 when it did occur in 2 if he goes into a detect state in 2 or if he does not go into a detect state and correctly guesses that it is a 2 . Thus

$$
\mathbf{P}(2 \mid 2)=\mathbf{P}(\mathrm{D} 2 \mid \mathrm{S} 2)+[(1-\mathbf{P}(\mathrm{D} 2 \mid \mathrm{S} 2)] \mathrm{G} 2 .
$$

He says the signal occurred in Observation Interval 1 when it was in 2 when he fails to go into D2 and incorrectly guesses:

$$
P 1 \mid 2)=[1-P(D 2 \mid S 2)] G 1 \text {. }
$$

To see that this model makes the decision threshold prediction for the frequency and loudness cue, let us write the probability correct as the sum of $1 / 2 \mathrm{P}(1 \mid 1)$ and $1 / 2 \mathbf{P}(2 \mid 2)$ :

$$
P(C)=1 / 2 P(1 \mid 1)+1 / 2 P(2 \mid 2) .
$$

(Type 1 and Type 2 trials occur each with probability $1 / 2$.) If we assume that the probability of going into a detect state, given a signal does not depend on which interval the subject is listening to, then we can let

$$
\mathrm{P}(\mathrm{D} 1 \mid \mathrm{S} 1)=\mathrm{P}(\mathrm{D} 2 \mid \mathrm{S} 2)=\mathrm{P}(\mathrm{D}) \text {. }
$$

Equations 9, 11, 13, and 14 let us write

$$
\begin{aligned}
P(C)=P(D) & +[1-P(D)]^{1 / 2}\{P(N 2 \mid S 2) \\
& +[1-P(N 2 \mid S 2)] G 1+G 2\},
\end{aligned}
$$

which allows the decision threshold prediction. In fact, Equation 15 is identical to Equation 6 if

$1 / 2\{P(N 2 \mid \mathrm{S} 2)+[1-\mathrm{P}(\mathrm{N} \mid 2 \mathrm{~S}) \mid \mathrm{G} 1+\mathrm{G} 2\}=.5$.

In order to test this model some other way, let us consider a situation in which we withhold information from one interval or the other. Normally, a Type 1 trial contains a signal in the first interval, and a Type 2 trial contains a signal in the second. When information is withheld from the first interval, a Type 1 trial will not have a signal in either Interval 1 or Interval 2, but a Type 2 trial will be a normal lype 2 trial, that is, a signal will be presented in the second observation interval. When information is withheld from the second observation interval, then a Type 1 trial is a normal trial with a signal in the first interval and a Type 2 trial does not have a signal in either interval.

When information is withheld from the first interval, $P(D 1)=0$, Equations 9 and 10 apply and become: 


$$
\begin{gathered}
\mathrm{P}(1 \mid 1)=\mathrm{P}(\mathrm{N} 2 \mid \overline{\mathrm{S}} 2)+[1-\mathrm{P}(\mathrm{N} 2 \mid \overline{\mathrm{S}} 2)] \mathrm{G} 1 \\
\mathrm{P}(2 \mid 1)=[1-\mathrm{P}(\mathrm{N} 2 \mid \mathrm{S} 2)] \mathrm{G} 2
\end{gathered}
$$

When information is withheld from the second observation interval, Equations 11 and 12 are affected and they become

$$
\begin{aligned}
& P(2 \mid 2)=G 2 \\
& P(1 \mid 2)=G 1 .
\end{aligned}
$$

Thus, performance on Type 2 trials is reduced to guessing when information is withheld from the second interval, but performance on Type 1 interval is not reduced to guessing when information is withheld from the first interval.

\section{EXPERIMENT II}

To test these predictions, one of the subjects was called back and run in each of three conditions. One condition was a normal 2ATFC experiment; another (B1) was one in which information was withheld in the first interval; and in the third (B2), information was withheld from the second interval. This signal was a frequency shift of $5 \mathrm{~Hz}$ together with an intensity increment of $2 \mathrm{~dB}$. Thus, when information was withheld from the first interval, neither frequency shift nor intensity increment was permitted in the first observation interval; and similarly for the second interval. The subject was run for 1,800 trials in each condition. Every other aspect of this experiment was similar to the first experiment.

\section{Results and Discussion}

Table 2 shows the four conditional probabilities observed under the three conditions: normal 2ATFC, information withheld from the first interval (B1), and information withheld from the second interval (B2). We see that the predictions are qualitatively substantiated. Performance on the Type 2 trials is not affected when information is withheld from the first interval, but performance on Type 1 trials is reduced although it has not dropped to chance. However, performance on Type 2 trials is drastically affected when information is withheld from the second interval and appears to be at approximately the chance level. These results are consistent with Campbell's (1969) findings that subjects seem to listen only to the first interval in a two-interval adaptive threshold paradigm.

The model is not put forward as an exact representation of the decision process in $2 \mathrm{ATFC}$ experiments, but rather as a heuristic indicative, perhaps, of a class of models which can account for the decision threshold results of the first experiment.
Table 2

Conditional Probabilities in Each of the Three Conditions

\begin{tabular}{lcccc}
\hline Condition & $P(111)$ & $P(112)$ & $P(212)$ & $P(112)$ \\
\hline Normal & .862 & .136 & .892 & .108 \\
B1 & .735 & .265 & .900 & .100 \\
B2 & .800 & .200 & .448 & .552 \\
\hline
\end{tabular}

It shows how perceptual independence can operate for loudness and pitch in a 2ATFC experiment and suggests a sequential decision process in this kind of experiment.

If, indeed, subjects use a sequential decision strategy in the two-interval experiment, the probability correct in this experiment cannot be interpreted as the area under the receiver operating characteristic. The area under the receiver operating characteristic has the rather nice property of being a measure of sensitivity which does not depend on the subject's criterion or upon the distributions associated with noise and signal plus noise. Thus, any conclusions drawn from the 2ATFC experiment which rely on these properties must be called into question.

\section{CONCLUSION}

To return to the problem of representing stimulus similarity, we might ask, "What implication do these data have concerning the spatial representation of stimulus similarity?"

Firstly, subjects are able to use both dimensions in this task. This is unlike the results of Shepard (1964) and Zagorski (Note 1), in which the subjects seemed to use only one dimension or the other. The study presented here clearly indicates that the subject can use both pitch and loudness during the same short time period.

Secondly, because of the fit to decision threshold model we can say that these data suggest that pitch information and loudness information are not combined. This is a surprise from Torgerson's (1958), Shepard's (1964), and Hyman and Well's (1967) point of view that dimensions are combined for nonanalyzable stimuli. Sinusoidal tones are nonanalyzable in the sense that one cannot present a pitch that has no loudness or a loudness that has no pitch.

Thirdly, insofar as the information integration model is consistent with a Euclidean metric representation of stimulus similarity (the $d^{\prime}$ in the information integration model is a Euclidean metric), these results surprise the specific expectation expressed by Torgerson. Shepard, and Hyman and Well that the Euclidean metric would work very well for such stimuli as sinusoidal tones.

Returning to the problem of subjects' strategies in 2ATFC tasks, neither the signal detection theoretic interpretation of this task nor Treisman and 
Leshowitz's (1969) differencing model or their double detection model fit the data presented here. Further examination of behavior in 2ATFC experiments would be in order.

\section{REFERENCE NOTE}

1. Zakorski. M. A. Some necessary conditions for the spatial representations of stimulus similarity. Mathematical Psychology Meeting, University of Michigan-Ann Arbor, 1969.

\section{REFERENCES}

Campbell, F. A. Context and sequence effects with an adaptive threshold procedure. Journal of the Acoustical Society of America, 1969, 46, 350-355.

Corcoran, D. W. J. Perceptual independence and recognition of two-dimensional auditory stimuli. Journal of the Acoustical Society of America, 1967, 42, 139-142.

Corcoran, D. W. J. Pattern recognition. Middlesex, England: Penguin, 1961.

GrEEN, D. M., \& SwETS, J. A. Signal detection theory and psychophysics. New York: Wiley, 1966.

Harris, J. D., Pickler, A. G., Hoffman, H. S., \& Ehmer, R. H. The interaction of pitch and loudness discriminations. Journal of Experimental Psychology, 1958, 56, 232-238.

HymaN, R., \& WELL, A. Judgements of similarity and spatial models. Perception \& Psychophysics, 1967, 2, 233-248.
Jauhiainen, T., HäkKiene, V., Lindroos, R., \& RaiJ, K. On pitch and loudness interaction. Journal of Auditory Research, 1967. 7. 41.46 .

Pollack, I. On the combination of intensity and frequency differences in auditory discrimination. Journal of the Acoustical Society of America, 1961, 33, 1141-1142.

REENPÄ̈, Y. Theorie des Sinneswahrnehmens. Annales Academiue Scientiarum Fennicae. Series A, V Medica, 1961, 78.

ShePard. R. N. Attention and the metric structures of the stimulus space. Journal of Mathematical Psychology, 1964, 1, 54-87.

TANNER, W. P., JR. Theory of recognition. Journal of the Acoustical Society of America, 1956, 28, 882-838.

TORGERson, W. Theory and methods of scaling. New York: Wiley, 1958.

Treisman, M., \& Leshowitz, B. The effects of duration, area, and background intensity on the visual intensity difference threshold given by the forced choice procedure: Derivations from a statistical decision model for sensory discrimination. Perception \& Psychophysics, 1969, 6, 281-296.

\section{NOTE}

1. The entire experiment was run and analyzed using the IBM 1800 process controller in the Mathematical Psychology Laboratory at Indiana University.

(Received for publication May 3, 1974; revision received October 2,1974 .) 